VOL. 73 (2006) [17-26]

\title{
SOME SCHWARZ TYPE INEQUALITIES FOR SEQUENCES OF OPERATORS IN HILBERT SPACES
}

\author{
SeVER S. DRAgomir
}

We give some inequalities of Cauchy-Bunyakovsky-Schwarz type for sequences of bounded linear operators in Hilbert spaces with applications.

\section{INTRODUCTION}

Let $(H ;(\cdot, \cdot))$ be a real or complex Hilbert space and $B(H)$ the Banach algebra of all bounded linear operators that map $H$ into $H$.

We recall that a self-adjoint operator $A \in B(H)$ is positive in $B(H)$ if and only if $(A x, x) \geqslant 0$ for any $x \in H$. The binary relation $A \geqslant B$ if and only if $A-B$ is a positive self-adjoint operator, is an order relation on $B(H)$. We remark that for any $A \in B(H)$ the operators $U:=A A^{*}$ and $V:=A^{*} A$ are positive self adjoint operators on $H$ and $\|U\|=\|V\|=\|A\|^{2}$.

In [1], the author has proved the following inequality of Cauchy-BunyakovskySchwarz type in the order of $B(H)$.

Theorem 1. Let $A_{1}, \ldots, A_{n} \in B(H)$ and $z_{1}, \ldots, z_{n} \in \mathbb{K}(\mathbb{R}, \mathbb{C})$. Then the following inequality holds:

$$
\sum_{i=1}^{n}\left|z_{i}\right|^{2} \sum_{i=1}^{n} A_{i} A_{i}^{*} \geqslant\left(\sum_{i=1}^{n} z_{i} A_{i}\right)\left(\sum_{i=1}^{n} \overline{z_{i}} A_{i}^{*}\right) \geqslant 0 .
$$

Proof: For the sake of completeness, we give here a simple proof of this inequality. For any $i, j \in\{1, \ldots, n\}$ one has in the order of $B(H)$ :

$$
\left(\overline{z_{i}} A_{j}-\overline{z_{j}} A_{i}\right)\left(\overline{z_{i}} A_{j}-\overline{z_{j}} A_{i}\right)^{*} \geqslant 0
$$

that is,

$$
\left(\overline{z_{i}} A_{j}-\overline{z_{j}} A_{i}\right)\left(z_{i} A_{j}^{*}-z_{j} A_{i}^{*}\right) \geqslant 0
$$

where

$$
\left|z_{i}\right|^{2} A_{j} A_{j}^{*}+\left|z_{j}\right|^{2} A_{i} A_{i}^{*} \geqslant \overline{z_{i}} z_{j} A_{j} A_{i}^{*}+\overline{z_{j}} z_{i} A_{i} A_{j}^{*}
$$

Received 4th July, 2005

Copyright Clearance Centre, Inc. Serial-fee code: 0004-9727/06 \$A2.00+0.00. 
for any $i, j \in\{1, \ldots, n\}$.

If we sum (1.2) over $i$ from 1 to $n$ we deduce

$$
\left(\sum_{i=1}^{n}\left|z_{i}\right|^{2}\right) A_{j} A_{j}^{*}+\left|z_{j}\right|^{2}\left(\sum_{i=1}^{n} A_{i} A_{i}^{*}\right) \geqslant z_{j} A_{j}\left(\sum_{i=1}^{n} \overline{z_{i}} A_{i}^{*}\right)+\left(\sum_{i=1}^{n} z_{i} A_{i}\right) \overline{z_{j}} A_{j}^{*}
$$

for any $j \in\{1, \ldots, n\}$.

If we sum (1.3) over $j$ from 1 to $n$, we deduce

$$
\begin{aligned}
\sum_{i=1}^{n}\left|z_{i}\right|^{2} \sum_{j=1}^{n} A_{j} A_{j}^{*}+\sum_{j=1}^{n}\left|z_{j}\right|^{2}\left(\sum_{i=1}^{n} A_{i} A_{i}^{*}\right) & \\
& \geqslant \sum_{j=1}^{n} z_{j} A_{j} \sum_{i=1}^{n} \overline{z_{i}} A_{i}^{*}+\left(\sum_{i=1}^{n} z_{i} A_{i}\right)\left(\sum_{j=1}^{n} \overline{z_{j}} A_{j}^{*}\right),
\end{aligned}
$$

that is,

$$
\sum_{k=1}^{n}\left|z_{k}\right|^{2} \sum_{k=1}^{n} A_{k} A_{k}^{*} \geqslant \sum_{k=1}^{n} z_{k} A_{k} \sum_{k=1}^{n} \overline{z_{k}} A_{k}^{*}=\left(\sum_{k=1}^{n} z_{k} A_{k}\right)\left(\sum_{k=1}^{n} z_{k} A_{k}\right)^{*} \geqslant 0,
$$

and the theorem is proved.

The following version of the Cauchy-Bunyakovsky-Schwarz inequality for norms also holds [1].

COROLlary 1. With the assumptions in Theorem 1, one has

$$
\sum_{k=1}^{n}\left|z_{k}\right|^{2}\left\|\sum_{k=1}^{n} A_{k} A_{k}^{*}\right\| \geqslant\left\|\sum_{k=1}^{n} z_{k} A_{k}\right\|^{2}
$$

Proof: The operators:

$$
A:=\sum_{k=1}^{n}\left|z_{k}\right|^{2} \sum_{k=1}^{n} A_{k} A_{k}^{*}, \quad B:=\left(\sum_{k=1}^{n} z_{k} A_{k}\right)\left(\sum_{k=1}^{n} \overline{z_{k}} A_{k}^{*}\right)
$$

are obviously self-adjoint, positive and by (1.1), $A \geqslant B \geqslant 0$. Thus $\|A\| \geqslant\|B\|$ and since,

$$
\|A\|=\sum_{k=1}^{n}\left|z_{k}\right|^{2}\left\|\sum_{k=1}^{n} A_{k} A_{k}^{*}\right\|
$$

and

$$
\|B\|=\left\|\sum_{k=1}^{n} z_{k} A_{k}\right\|^{2}
$$

the corollary is proved.

For other related results, see [2].

The main aim of this paper is to point out other inequalities similar to (1.6). 


\section{NORM INEQUALITIES}

The following result holds.

TheOREM 2. Let $\alpha_{1}, \ldots, \alpha_{n} \in \mathbb{K}$ and $A_{1}, \ldots, A_{n} \in B(H)$. Then one has the inequalities:

$$
\begin{aligned}
\left\|\sum_{i=1}^{n} \alpha_{i} A_{i}\right\|^{2} \leqslant\left\{\begin{array}{l}
\max _{i=1, n}\left|\alpha_{i}\right|^{2} \sum_{i=1}^{n}\left\|A_{i}\right\|^{2} \\
\left(\sum_{i=1}^{n}\left|\alpha_{i}\right|^{2 p}\right)^{1 / p}\left(\sum_{i=1}^{n}\left\|A_{i}\right\|^{2 q}\right)^{1 / q} \text { if } p>1, \frac{1}{p}+\frac{1}{q}=1 ; \\
\sum_{i=1}^{n}\left|\alpha_{i}\right|^{2} \max _{i=1, n}\left\|A_{i}\right\|^{2}
\end{array}\right. \\
+\left\{\begin{array}{l}
\max _{1 \leqslant i \neq j \leqslant n}\left\{\left|\alpha_{i}\right|\left|\alpha_{j}\right|\right\} \sum_{1 \leqslant i \neq j \leqslant n}\left\|A_{i} A_{j}^{*}\right\| \\
{\left[\left(\sum_{i=1}^{n}\left|\alpha_{i}\right|^{r}\right)^{2}-\sum_{i=1}^{n}\left|\alpha_{i}\right|^{2 r}\right]^{1 / r}\left(\sum_{1 \leqslant i \neq j \leqslant n}\left\|A_{i} A_{j}^{*}\right\|^{s}\right)^{1 / s}} \\
\text { if } r>1, \frac{1}{r}+\frac{1}{s}=1 ; \\
{\left[\left(\sum_{i=1}^{n}\left|\alpha_{i}\right|\right)^{2}-\sum_{i=1}^{n}\left|\alpha_{i}\right|^{2}\right] \max _{1 \leqslant i \neq j \leqslant n}\left\|A_{i} A_{j}^{*}\right\|,}
\end{array}\right.
\end{aligned}
$$

where (2.1) should be seen as all the 9 possible configurations.

Proof: We have

$$
\begin{aligned}
0 & \leqslant\left(\sum_{i=1}^{n} \alpha_{i} A_{i}\right)\left(\sum_{i=1}^{n} \alpha_{i} A_{i}\right)^{*}=\left(\sum_{i=1}^{n} \alpha_{i} A_{i}\right)\left(\sum_{j=1}^{n} \overline{\alpha_{j}} A_{j}^{*}\right) \\
& =\sum_{i=1}^{n} \sum_{j=1}^{n} \alpha_{i} \overline{\alpha_{j}} A_{i} A_{j}^{*}=\sum_{i=1}^{n}\left|\alpha_{i}\right|^{2} A_{i} A_{i}^{*}+\sum_{1 \leqslant i \neq j \leqslant n} \alpha_{i} \overline{\alpha_{j}} A_{i} A_{j}^{*} .
\end{aligned}
$$

Taking the norm in (2.2) and observing that $\left\|U U^{*}\right\|=\|U\|^{2}$ for any $U \in B(H)$, one has the inequality

$$
\begin{aligned}
\left\|\sum_{i=1}^{n} \alpha_{i} A_{i}\right\|^{2} & =\left\|\sum_{i=1}^{n}\left|\alpha_{i}\right|^{2} A_{i} A_{i}^{*}+\sum_{1 \leqslant i \neq j \leqslant n} \alpha_{i} \overline{\alpha_{j}} A_{i} A_{j}^{*}\right\| \\
& \leqslant \sum_{i=1}^{n}\left|\alpha_{i}\right|^{2}\left\|A_{i} A_{i}^{*}\right\|+\sum_{1 \leqslant i \neq j \leqslant n}\left|\alpha_{i}\right|\left|\alpha_{j}\right|\left\|A_{i} A_{j}^{*}\right\| \\
& =\sum_{i=1}^{n}\left|\alpha_{i}\right|^{2}\left\|A_{i}\right\|^{2}+\sum_{1 \leqslant i \neq j \leqslant n}\left|\alpha_{i}\right|\left|\alpha_{j}\right|\left\|A_{i} A_{j}^{*}\right\| .
\end{aligned}
$$


Using Hölder's inequality, we may write that:

$$
\sum_{i=1}^{n}\left|\alpha_{i}\right|^{2}\left\|A_{i}\right\|^{2} \leqslant\left\{\begin{array}{l}
\max _{i=\overline{1, n}}\left|\alpha_{i}\right|^{2} \sum_{i=1}^{n}\left\|A_{i}\right\|^{2} \\
\left(\sum_{i=1}^{n}\left|\alpha_{i}\right|^{2 p}\right)^{1 / p}\left(\sum_{i=1}^{n}\left\|A_{i}\right\|^{2 q}\right)^{1 / q} \text { if } p>1, \frac{1}{p}+\frac{1}{q}=1 \\
\sum_{i=1}^{n}\left|\alpha_{i}\right|^{2} \max _{i=1, n}\left\|A_{i}\right\|^{2}
\end{array}\right.
$$

Also, Hölder's inequality for double sums produces

$$
\sum_{1 \leqslant i \neq j \leqslant n}\left|\alpha_{i}\right|\left|\alpha_{j}\right|\left\|A_{i} A_{j}^{*}\right\| \leqslant\left\{\begin{array}{l}
\max _{1 \leqslant i \neq j \leqslant n}\left\{\left|\alpha_{i}\right|\left|\alpha_{j}\right|\right\} \sum_{1 \leqslant i \neq j \leqslant n}\left\|A_{i} A_{j}^{*}\right\| \\
\left.\left(\sum_{1 \leqslant i \neq j \leqslant n}\left|\alpha_{i}\right|^{r}\left|\alpha_{j}\right|^{r}\right)^{1 / r} \sum_{1 \leqslant i \neq j \leqslant n}\left\|A_{i} A_{j}^{*}\right\|^{s}\right)^{1 / s} \\
\text { if } r>1, \frac{1}{r}+\frac{1}{s}=1 ; \\
\sum_{1 \leqslant i \neq j \leqslant n}\left|\alpha_{i}\right|\left|\alpha_{j}\right| \max _{1 \leqslant i \neq j \leqslant n}\left\|A_{i} A_{j}^{*}\right\|, \\
=\left\{\begin{array}{l}
\max _{1 \leqslant i \neq j \leqslant n}\left\{\left|\alpha_{i}\right|\left|\alpha_{j}\right|\right\} \sum_{1 \leqslant i \neq j \leqslant n}\left\|A_{i} A_{j}^{*}\right\| \\
\left.\left[\left(\sum_{i=1}^{n}\left|\alpha_{i}\right|^{r}\right)^{2}-\sum_{i=1}^{n}\left|\alpha_{i}\right|^{2 r}\right]_{1 \leqslant i \neq j} \sum_{1 \leqslant n}\left\|A_{i} A_{j}^{*}\right\|^{s}\right)^{1 / s} \\
{\left[\left(\sum_{i=1}^{n}\left|\alpha_{i}\right|\right)^{2}-\sum_{i=1}^{n}\left|\alpha_{i}\right|^{2}\right]_{1 \leqslant i \neq j \leqslant n}+\frac{1}{s}=1 ;}
\end{array}\right.
\end{array}\right.
$$

Using (2.3) and (2.4), (2.5) one deduces the desired inequality (2.1).

The following corollaries are natural consequences.

COROLLARY 2. With the assumptions of Theorem 2, one has the inequality

$$
\left\|\sum_{i=1}^{n} \alpha_{i} A_{i}\right\| \leqslant \max _{i=\overline{1, n}}\left|\alpha_{i}\right|\left(\sum_{i, j=1}^{n}\left\|A_{i} A_{j}^{*}\right\|\right)^{1 / 2}
$$

Proof: Follows by the first line in (2.1) on taking into account that

$$
\max _{1 \leqslant i \neq j \leqslant n}\left\{\left|\alpha_{i}\right|\left|\alpha_{j}\right|\right\} \leqslant \max _{i=\overline{1, n}}\left|\alpha_{i}\right|^{2}
$$

and

$$
\sum_{i, j=1}^{n}\left\|A_{i} A_{j}^{*}\right\|=\sum_{i=1}^{n}\left\|A_{i}\right\|^{2}+\sum_{1 \leqslant i \neq j \leqslant n}\left\|A_{i} A_{j}^{*}\right\|
$$


Corollary 3. With the assumptions in Theorem 2, one has the inequality:

$$
\begin{aligned}
& \left\|\sum_{i=1}^{n} \alpha_{i} A_{i}\right\|^{2} \\
& \quad \leqslant\left(\sum_{i=1}^{n}\left|\alpha_{i}\right|^{2 p}\right)^{1 / p}\left[\left(\sum_{i=1}^{n}\left\|A_{i}\right\|^{2 q}\right)^{1 / q}+(n-1)^{1 / p}\left(\sum_{1 \leqslant i \neq j \leqslant n}\left\|A_{i} A_{j}^{*}\right\|^{q}\right)^{1 / q}\right],
\end{aligned}
$$

where $p>1,1 / p+1 / q=1$.

PROOF: Using the Cauchy-Bunyakovsky-Schwarz inequality for positive numbers

$$
\left(\sum_{i=1}^{n} a_{i}\right)^{2} \leqslant n \sum_{i=1}^{n} a_{i}^{2}
$$

we may write that

$$
\begin{aligned}
\left(\sum_{i=1}^{n}\left|\alpha_{i}\right|^{p}\right)^{2}-\sum_{i=1}^{n}\left|\alpha_{i}\right|^{2 p} & \leqslant n \sum_{i=1}^{n}\left|\alpha_{i}\right|^{2 p}-\sum_{i=1}^{n}\left|\alpha_{i}\right|^{2 p} \\
& =(n-1) \sum_{i=1}^{n}\left|\alpha_{i}\right|^{2 p}
\end{aligned}
$$

Now, using the second line in (2.1) for $r=p, s=q$, we deduce the desired result (2.7).

Corollary 4. With the assumptions in Theorem 2, one has the inequality

$$
\left\|\sum_{i=1}^{n} \alpha_{i} A_{i}\right\|^{2} \leqslant \sum_{i=1}^{n}\left|\alpha_{i}\right|^{2}\left[\max _{i=\overline{1, n}}\left\|A_{i}\right\|^{2}+(n-1) \max _{1 \leqslant i \neq j \leqslant n}\left\|A_{i} A_{j}^{*}\right\|\right] .
$$

ProOF: Follows by the third line of (2.1) on taking into account that

$$
\left(\sum_{i=1}^{n}\left|\alpha_{i}\right|\right)^{2}-\sum_{i=1}^{n}\left|\alpha_{i}\right|^{2} \leqslant(n-1) \sum_{i=1}^{n}\left|\alpha_{i}\right|^{2}
$$

Another interesting particular case is embodied in the following corollary as well.

COROLlary 5. With the assumptions in Theorem 2, one has the inequality

$$
\left\|\sum_{i=1}^{n} \alpha_{i} A_{i}\right\|^{2} \leqslant \sum_{i=1}^{n}\left|\alpha_{i}\right|^{2}\left[\max _{i=1, n}\left\|A_{i}\right\|^{2}+\left(\sum_{1 \leqslant i \neq j \leqslant n}\left\|A_{i} A_{j}^{*}\right\|^{2}\right)^{1 / 2}\right] .
$$

PROOF: It is obvious that

$$
\left[\left(\sum_{i=1}^{n}\left|\alpha_{i}\right|^{2}\right)^{2}-\sum_{i=1}^{n}\left|\alpha_{i}\right|^{4}\right]^{1 / 2} \leqslant \sum_{i=1}^{n}\left|\alpha_{i}\right|^{2} .
$$

Thus, combining the third line in the first bracket in (2.1) with the second line for $r=s \approx 2$ in the second bracket, the inequality (2.9) is obtained. 
REMARK 1. If one is interested in obtaining bounds in terms of $\sum_{i=1}^{n}\left|\alpha_{i}\right|^{2}$, there are other possibilities as shown below. Obviously, since

$$
\max _{1 \leqslant i \neq j \leqslant n}\left\{\left|\alpha_{i}\right|\left|\alpha_{j}\right|\right\} \leqslant \max _{i=1, n}\left|\alpha_{i}\right|^{2} \leqslant \sum_{i=1}^{n}\left|\alpha_{i}\right|^{2} .
$$

then, by $(2.1)$, in choosing the third line in the first bracket with the first line in the second bracket, one would obtain

$$
\left\|\sum_{i=1}^{n} \alpha_{i} A_{i}\right\|^{2} \leqslant \sum_{i=1}^{n}\left|\alpha_{i}\right|^{2}\left[\max _{i=1, n}\left\|A_{i}\right\|^{2}+\sum_{1 \leqslant i \neq j \leqslant n}\left\|A_{i} A_{j}^{*}\right\|\right] .
$$

Also, it is evident that

$$
\left[\left(\sum_{i=1}^{n}\left|\alpha_{i}\right|^{r}\right)^{2}-\sum_{i=1}^{n}\left|\alpha_{i}\right|^{2 r}\right]^{1 / r} \leqslant\left(\sum_{i=1}^{n}\left|\alpha_{i}\right|^{r}\right)^{2 / r}
$$

By the monotonicity of the power mean $\left(\left(\sum_{i=1}^{n} / n\right) a_{i}^{m}\right)^{1 / m}$ as a function of $m \in \mathbb{R}$, we have

$$
\left(\frac{\sum_{i=1}^{n}\left|\alpha_{i}\right|^{r}}{n}\right)^{1 / r} \leqslant\left(\frac{\sum_{i=1}^{n}\left|\alpha_{i}\right|^{2}}{n}\right)^{1 / 2}, \quad 1<r \leqslant 2
$$

giving

$$
\left(\sum_{i=1}^{n}\left|\alpha_{i}\right|^{r}\right)^{2 / r} \leqslant n^{2 / r-1} \sum_{i=1}^{n}\left|\alpha_{i}\right|^{2}
$$

Thus, using the third line in the first bracket of (2.1) combined with the second line in the second bracket for $1<r \leqslant 2,1 / s+1 / r=1$, we deduce

$$
\left\|\sum_{i=1}^{n} \alpha_{i} A_{i}\right\|^{2} \leqslant \sum_{i=1}^{n}\left|\alpha_{i}\right|^{2}\left[\max _{i=1, n}\left\|A_{i}\right\|^{2}+n^{(2 / r)-1}\left(\sum_{1 \leqslant i \neq j \leqslant n}\left\|A_{i} A_{j}^{*}\right\|^{s}\right)^{1 / s}\right] .
$$

Note that for $r=s=2$, we recapture (2.9).

The following particular result also holds.

Proposition 1. Let $\alpha_{1}, \ldots, \alpha_{n} \in \mathbb{K}$ and $A_{1}, \ldots, A_{n} \in B(H)$ with the property that $A_{i} A_{j}^{*}=0$ for any $i \neq j, i, j \in\{1, \ldots, n\}$. Then one has the inequality;

$$
\left\|\sum_{i=1}^{n} \alpha_{i} A_{i}\right\| \leqslant\left\{\begin{array}{l}
\max _{i=\overline{1, n}}\left|\alpha_{i}\right|\left(\sum_{i=1}^{n}\left\|A_{i}\right\|^{2}\right)^{1 / 2} \\
\left(\sum_{i=1}^{n}\left|\alpha_{i}\right|^{2 p}\right)^{1 /(2 p)}\left(\sum_{i=1}^{n}\left\|A_{i}\right\|^{2 q}\right)^{1 /(2 q)} \text { if } p>1, \frac{1}{p}+\frac{1}{q}=1 \\
\left(\sum_{i=1}^{n}\left|\alpha_{i}\right|^{2}\right)^{1 / 2} \max _{i=1, n}\left\|A_{i}\right\| .
\end{array}\right.
$$


If by $M(\alpha, \mathbf{A})$ we denote any of the bounds provided by $(2.1),(2.6),(2.7),(2.8)$, (2.9), (2.10) or (2.11), then we may state the following proposition as well.

Proposition 2. Under the assumptions of Theorem 2, we have:

(i) For any $x \in H$

$$
\left\|\sum_{i=1}^{n} \alpha_{i} A_{i} x\right\|^{2} \leqslant\|x\|^{2} M(\alpha, \mathbf{A}) .
$$

(ii) For any $x, y \in H$,

$$
\left|\sum_{i=1}^{n} \alpha_{i}\left\langle A_{i} x, y\right\rangle\right|^{2} \leqslant\|x\|^{2}\|y\|^{2} M(\alpha, \mathbf{A})
$$

ProOF:

(i) Obviously,

$$
\begin{aligned}
\left\|\sum_{i=1}^{n} \alpha_{i} A_{i} x\right\|^{2} & =\left\|\left(\sum_{i=1}^{n} \alpha_{i} A_{i}\right)(x)\right\|^{2} \leqslant\left\|\sum_{i=1}^{n} \alpha_{i} A_{i}\right\|^{2}\|x\|^{2} \\
& \leqslant M(\alpha, \mathbf{A})\|x\|^{2} .
\end{aligned}
$$

(ii) We have

$$
\left|\sum_{i=1}^{n} \alpha_{i}\left\langle A_{i} x, y\right\rangle\right|^{2}=\left|\left\langle\sum_{i=1}^{n} \alpha_{i} A_{i} x, y\right\rangle\right|^{2} \leqslant\left\|\sum_{i=1}^{n} \alpha_{i} A_{i} x\right\|^{2}\|y\|^{2},
$$

which, by (i), gives the desired result (2.14).

\section{Inequalities for Vectors in Hilbert Spaces}

We consider the non zero vectors $y_{1}, \ldots, y_{n} \in H$. Define the operators

$$
A_{i}: H \rightarrow H, \quad A_{i} x=\frac{\left(x, y_{i}\right)}{\left\|y_{i}\right\|} \cdot y_{i}, \quad i \in\{1, \ldots, n\}
$$

Since

$$
\left\|A_{i}\right\|=\sup _{\|x\|=1}\left\|A_{i} x\right\|=\sup _{\|x\|=1}\left|\left(x, y_{i}\right)\right|=\left\|y_{i}\right\|, \quad i \in\{1, \ldots, n\}
$$

then $A_{i}$ are bounded linear operators in $H$. Also, since

$$
\left(A_{i} x, x\right)=\left(\frac{\left(x, y_{i}\right) y_{i}}{\left\|y_{i}\right\|}, x\right)=\frac{\left|\left(x, y_{i}\right)\right|^{2}}{\left\|y_{i}\right\|} \geqslant 0, \quad x \in H, \quad i \in\{1, \ldots, n\}
$$


and

$$
\begin{aligned}
& \left(A_{i} x, z\right)=\left(\frac{\left(x, y_{i}\right) y_{i}}{\left\|y_{i}\right\|}, z\right)=\frac{\left(x, y_{i}\right)\left(y_{i}, z\right)}{\left\|y_{i}\right\|} \\
& \left(x, A_{i} z\right)=\left(x, \frac{\left(z, y_{i}\right) y_{i}}{\left\|y_{i}\right\|}\right)=\frac{\left(x, y_{i}\right)\left(z, y_{i}\right)}{\left\|y_{i}\right\|}=\frac{\left(x, y_{i}\right)\left(y_{i}, z\right)}{\| y_{i}}
\end{aligned}
$$

giving

$$
\left(A_{i} x, z\right)=\left(x, A_{i} z\right), \quad x, z \in H, \quad i \in\{1, \ldots, n\},
$$

we may conclude that $A_{i}(i=1, \ldots, n)$ are positive self-adjoint operators on $H$.

Since, for any $x \in H$, one has

$$
\begin{aligned}
\left\|\left(A_{i} A_{j}\right)(x)\right\| & =\left\|\left(A_{i}\right)\left(A_{j} x\right)\right\|=\left\|A_{i}\left(\frac{\left(x, y_{j}\right) y_{j}}{\left\|y_{j}\right\|}\right)\right\| \\
& =\frac{\left|\left(x, y_{j}\right)\right|}{\left\|y_{j}\right\|}\left\|A_{i} y_{j}\right\|=\frac{\left|\left(x, y_{j}\right)\right|}{\left\|y_{j}\right\|} \cdot \frac{\mid\left(y_{j}, y_{i}\right)\left\|y_{j}\right\|}{\left\|y_{j}\right\|} \\
& =\frac{\left|\left(x, y_{j}\right) \|\right|\left(y_{j}, y_{i}\right) \mid}{\left\|y_{j}\right\|}, \quad i, j \in\{1, \ldots, n\},
\end{aligned}
$$

we deduce that

$$
\left\|A_{i} A_{j}\right\|=\sup _{\|x\|=1} \frac{\left|\left(x, y_{j}\right) \|\left(y_{j}, y_{i}\right)\right|}{\left\|y_{j}\right\|}=\left|\left(y_{i}, y_{j}\right)\right| ; \quad i, j \in\{1, \ldots, n\} .
$$

If $\left(y_{i}\right)_{i=1, n}$ is an orthogonal family on $H$, then $\left\|A_{i}\right\|=1$ and $A_{i} A_{j}=0$ for $i, j \in\{1, \ldots, n\}$, $i \neq j$.

The following inequality for vectors holds.

THEOREM 3. Let $x, y_{1}, \ldots, y_{n} \in H$ and $\alpha_{n}, \ldots, \alpha_{n} \in \mathbb{R}$. Then one has the inequalities:

$$
\begin{aligned}
& \left\|\sum_{i=1}^{n} \alpha_{i} \frac{\left(x, y_{i}\right)}{\left\|y_{i}\right\|} y_{i}\right\|^{2} \\
& \quad \leqslant\|x\|^{2} \times\left\{\begin{array}{l}
\max _{i=1, n}\left|\alpha_{i}\right|^{2} \sum_{i=1}^{n}\left\|y_{i}\right\|^{2} \\
\left(\sum_{i=1}^{n}\left|\alpha_{i}\right|^{2 p}\right)^{1 / p}\left(\sum_{i=1}^{n}\left\|y_{i}\right\|^{2 q}\right)^{1 / q} \text { if } p>1, \frac{1}{p}+\frac{1}{q}=1 \\
\sum_{i=1}^{n}\left|\alpha_{i}\right|^{2} \max _{i=1, n}\left\|y_{i}\right\|^{2}
\end{array}\right.
\end{aligned}
$$




$$
+\|x\|^{2} \times\left\{\begin{array}{l}
\max _{1 \leqslant i \neq j \leqslant n}\left\{\left|\alpha_{i}\right|\left|\alpha_{j}\right|\right\} \sum_{1 \leqslant i \neq j \leqslant n}\left|\left(y_{i}, y_{j}\right)\right| \\
{\left[\left(\sum_{i=1}^{n}\left|\alpha_{i}\right|^{r}\right)^{2}-\sum_{i=1}^{n}\left|\alpha_{i}\right|^{2 r}\right]^{1 / r}\left(\sum_{1 \leqslant i \neq j \leqslant n}\left|\left(y_{i}, y_{j}\right)\right|^{s}\right)^{1 / s}} \\
\text { if } r>1, \frac{1}{r}+\frac{1}{s}=1 ; \\
{\left[\left(\sum_{i=1}^{n}\left|\alpha_{i}\right|\right)^{2}-\sum_{i=1}^{n}\left|\alpha_{i}\right|^{2}\right] \max _{1 \leqslant i \neq j \leqslant n}\left|\left(y_{i}, y_{j}\right)\right| .}
\end{array}\right.
$$

Proof: Follows by Theorem 2 and Proposition 2, (i) on choosing $A_{i}=\left(\left(\cdot, y_{i}\right) /\left\|y_{i}\right\|\right) y_{i}$ and taking into account that $\left\|A_{i}\right\|=\left\|y_{i}\right\|$,

$$
\left\|A_{i} A_{j}^{*}\right\|=\left|\left(y_{i}, y_{j}\right)\right|, \quad i, j \in\{1, \ldots, n\} .
$$

We omit the details.

Using Corollaries 2-5 and Remark 1, we may state the following particular inequalities:

$$
\begin{aligned}
& \left\|\sum_{i=1}^{n} \alpha_{i} \frac{\left(x, y_{i}\right)}{\left\|y_{i}\right\|} y_{i}\right\| \leqslant\|x\| \max _{i=1, n}\left|\alpha_{i}\right|\left(\sum_{i, j=1}^{n}\left|\left(y_{i}, y_{j}\right)\right|\right)^{1 / 2} ; \\
& \left\|\sum_{i=1}^{n} \alpha_{i} \frac{\left(x, y_{i}\right)}{\left\|y_{i}\right\|} y_{i}\right\|^{2} \leqslant\|x\|^{2}\left[( \sum _ { i = 1 } ^ { n } | \alpha _ { i } | ^ { 2 p } ) ^ { 1 / p } \left[\left(\sum_{i=1}^{n}\left\|y_{i}\right\|^{2 q}\right)^{1 / q}\right.\right. \\
& \left.\left.+(n-1)^{1 / p}\left(\sum_{1 \leqslant i \neq j \leqslant n}\left|\left(y_{i}, y_{j}\right)\right|^{q}\right)^{1 / q}\right]\right],
\end{aligned}
$$

where $p>1,1 / p+1 / q=1$

$$
\begin{aligned}
& \text { (3.8) }\left\|\sum_{i=1}^{n} \alpha_{i} \frac{\left(x, y_{i}\right)}{\left\|y_{i}\right\|} y_{i}\right\|^{2} \leqslant\|x\|^{2} \sum_{i=1}^{n}\left|\alpha_{i}\right|^{2}\left[\max _{i=1, n}\left\|y_{i}\right\|^{2}+(n-1) \max _{1 \leqslant i \neq j \leqslant n}\left|\left(y_{i}, y_{j}\right)\right|\right] ; \\
& \text { (3.9) }\left\|\sum_{i=1}^{n} \alpha_{i} \frac{\left(x, y_{i}\right)}{\left\|y_{i}\right\|} y_{i}\right\|^{2} \leqslant\|x\|^{2} \sum_{i=1}^{n}\left|\alpha_{i}\right|^{2}\left[\max _{i=\overline{1, n}}\left\|y_{i}\right\|^{2}+\left(\sum_{1 \leqslant i \neq j \leqslant n}\left|\left(y_{i}, y_{j}\right)\right|^{2}\right)^{1 / 2}\right] ; \\
& \text { (3.10) }\left\|\sum_{i=1}^{n} \alpha_{i} \frac{\left(x, y_{i}\right)}{\left\|y_{i}\right\|} y_{i}\right\|^{2} \leqslant\|x\|^{2} \sum_{i=1}^{n}\left|\alpha_{i}\right|^{2}\left[\max _{i=1, n}\left\|y_{i}\right\|^{2}+\sum_{1 \leqslant i \neq j \leqslant n}\left|\left(y_{i}, y_{j}\right)\right|\right] ; \\
& \text { (3.11) }\left\|\sum_{i=1}^{n} \alpha_{i} \frac{\left(x, y_{i}\right)}{\left\|y_{i}\right\|} y_{i}\right\|^{2} \leqslant\|x\|^{2} \sum_{i=1}^{n}\left|\alpha_{i}\right|^{2}\left[\max _{i=1, n}\left\|y_{i}\right\|^{2}+n^{(2 / r)-1}\left(\sum_{1 \leqslant i \neq j \leqslant n}\left|\left(y_{i}, y_{j}\right)\right|^{s}\right)^{1 / s}\right],
\end{aligned}
$$

where $1<r \leqslant 2,1 / s+1 / r=1$.

REMARK 2. The choice $\alpha_{i}=\left\|y_{i}\right\|(i=1, \ldots, n)$ will produce some interesting bounds for

$$
\left\|\sum_{i=1}^{n}\left(x, y_{i}\right) y_{i}\right\|^{2} \text {. }
$$

We omit the details. 


\section{REFERENCES}

[1] S.S. Dragomir, 'On some operatorial inequalities in Hilbert spaces', Bul. Stiinţ. Inst. Politehn. Cluj-Napoca Ser. Mat. Mec. Apl. Construc. mas. 30 (1987), 23-28.

[2] S.S. Dragomir and B. Mond, 'On Cauchy-Buniakowski-Schwarz's inequality for sequences of linear operators in Hilbert space', Houston J. Math. 22 (1996), 329-339.

School of Computer Science and Mathematics

Victoria University of Technology

PO Box 14428

MCMC 8001, Vic.

Australia

e-mail: sever@matilda.vu.edu.au 